Sports injury

\title{
The Western Australian sports injury study
}

\section{Stevenson, C Finch, P Hamer, B Elliott}

The magnitude and key risk and protective factors for sports injury at the population level in Australia have been established

W hile rates of regular physical activity are increasing among Australians, there is potential for an increase in the number of injuries. It is important therefore to consider the benefits attributed to participation in sport and recreational pursuits in the light of the increased physical risks. ${ }^{1}$

Until now, there has been a lack of valid, reliable, and recent data on the incidence and up to date costs of sports injuries in Australia. Furthermore, much of the research has focused on elite participants, ${ }^{2}$ with a paucity of research among non-elite sports participants, despite the fact that most sports participants play at a non-elite level. ${ }^{3}$ As the risk of injury and the concomitant rate of injury are likely to be different in elite and non-elite sports participants, there was a need for research that could be translated into injury prevention policy and practice for non-elite sports participants.

A proposal to establish the first Australian longitudinal population based study of sports injuries-the Western Australian sports injury study-was developed in collaboration with the peak non-government organisation for sports medicine in Australia-Sports Medicine Australia-and the authors. The broad aims of the study were to:

- determine the magnitude of the sports injury problem at the non-elite level of participation in Western Australia;

- determine the cost of sports injuries to the Western Australian community;

- provide an evidence base for the enhancement and upgrading of existing sports injury prevention and education programmes.

\section{STUDY DESIGN}

The study was designed to provide information about a large number of sports participants and their resultant injuries. Although this approach limits the detailed examination of specific clinical, anthropometric, and biomechanical influences on injury, it was capable of quantifying the key risk and protective factors for sports injury at a population level.

Cohort studies have been used to identify risk factors for injuries such as hip fractures and motor vehicle injury, ${ }^{4}$ but few longitudinal study designs have been undertaken to investigate the factors associated with sports injury risk, particularly at the community level. A cohort study design was therefore considered to be the most appropriate and robust methodology for achieving the study's objectives. The study design minimised the limitations of previous research especially in relation to the recall of injury events and the validity of self reported data and adjustment for player exposure, in both training sessions and games. These features, along with the excellent follow up of the participants over two consecutive winter sports seasons, ensured that the aims of the study were achieved.

A sample of 1512 participants, who played Australian football (33\% of sample), netball $(26 \%)$, field hockey $(29 \%)$, or basketball $(12 \%)$, was recruited from the Perth metropolitan area (Perth is the capital of the State of Western Australia with a population of 1.4 million). Participant recruitment reflected the way the sport is delivered in the state, hence player recruitment was through clubs and teams. To be eligible for recruitment, participants in selected teams needed to be competing at a non-elite level-that is, non-professional or first class competition-and to have not sustained a sports injury in the three months before the start of the study. A sports injury was defined as an injury that occurred during sports participation and led to one of the following consequences: a reduction in the amount or level of sports activity, the need for advice or treatment, and or adverse economic or social effects. ${ }^{5}$ Once recruited, the participants were followed up (by telephone) each month over two consecutive five month winter sports seasons.

Information was collected using a baseline self report questionnaire and monthly telephone interviews. The baseline questionnaire included items about the players' previous sports injury history, along with questions on their preseason training, warm up, and cool down patterns, specific items about the equipment used, such as footwear and protective devices, along with general health, lifestyle, and physical activity questions. The five-factor personality inventory test ${ }^{6}$ and the athletic life expectancy survey ${ }^{7}$ were also administered. The monthly telephone interview elicited whether the participant had sustained an injury in the preceding week, and, if so, the nature, extent, consequences and/or treatment received. Participants were also asked during the interview to recall the number of games and training sessions attended during the preceding four weeks. Extensive validation of self reported data including a comparison of medical records with self reported injuries was undertaken.

One of the aims of the study was to examine the cost of sports injury in Western Australia. Injury data collected as part of the study were used as the basis to estimate the cost of sports injury. Both the direct costs of treating injuries and the indirect costs resulting from time lost as a consequence of sustaining an injury were calculated. Based on the participation rates in Western Australia for each of the selected sports, the costs were extrapolated to a state level to provide estimates of the cost of injury to players of these sports. Further details on the study's design, methods (including statistical modelling), and findings are reported elsewhere. ${ }^{8-10}$

\section{THE FINDINGS}

The incidence of injury at the non-elite level of participation was 16 injuries per 1000 hours of sports participation, which is significantly less than the incidence of injury at the elite level of participation. As expected, the incidence of injury was highest in the contact sports such as Australian football (20/1000 hours) and lowest in netball (12/1000 hours). The injury incidence rate for hockey and basketball was 15 injuries per 1000 hours of participation. Injury incidence was significantly higher among male participants (19/1000 hours compared with 13/1000 hours) and in the 26-30 year old age group (20/1000 hours).

The likelihood of injury was reduced if participants had undertaken a training programme for their sport and more specifically, a programme designed by a sport's professional. In fact, the participants' risk of injury was reduced by $32 \%$ if they had undertaken a programme designed by a professional. The risk of injury was also reduced if the participants had played their sport in the 12 months before the start of the study. 
Experience from playing the specific sport in the past 12 months reduced the likelihood of injury by $29 \%$. Further protective factors against sports injury were not drinking alcohol ( $18 \%$ reduction), having high levels of endurance (10\% reduction), and being physically active beyond the sport ( $8 \%$ reduction).

\section{"A back problem increased the likelihood of injury by $69 \%$."}

The most significant independent predictor of sports injury was having a back problem that had been confirmed by a health professional. A back problem increased the likelihood of injury by $69 \%$. This finding was apparent after taking account of the age and sex of the player as well as the sport code they played. As might be expected, having sustained a sports injury in the 12 months preceding the study also significantly increased the likelihood of injury. The risk of injury was $45 \%$ higher in participants who had a previous injury compared with participants who had no prior injury.

An interesting finding was the identification of psychological variables as predictors of injury. Participants who rated themselves extroverted and open were also at a greater risk of injury than those who did not consider themselves thus.

The average cost of sports injury each season for participants in this study was AUD\$126 per injured player. This figure is dependent on the healthcare funding system in Australia so it will be an underestimate and may not be useful for non-Australians. This results in a total cost to Western Australia, for participants playing these four sports, of approximately AUD\$4.85 million per season.

\section{SUMMARY}

The Western Australian sports injury study has elucidated the magnitude and key risk and protective factors for sports injury at the population level in Australia for the first time. It has also provided an estimate of the costs associated with sports injury. As the first longitudinal study of sports injuries in Australia and one of only a few longitudinal studies internationally, it provides a platform for establishing a strategic direction for sports injury prevention, education, and policy in Western Australia and nationally.

As highlighted in the findings, sports injuries cost the community a sizeable amount, and cost savings could be achieved if effective preventive programmes are developed and implemented to reduce the incidence and severity of injury. Scientific evidence is the tenet upon which sports injury prevention strategies must be based, and this study, by identifying the key risk and protective factors for sports injury, has provided a focus for Sports Medicine Australia to develop new, and enhance established, sports injury prevention strategies. Furthermore, the study has provided data for evaluation of preventive strategies. Since the Western Australian sports injury study was undertaken, a further research project has been initiated, which aims to integrate the findings from the Western Australian sports injury study with research findings from time-motion analyses and a raft of other research projects in order to provide sufficient evidence to draft policies and best practices for reducing the incidence of injury in sport.

\section{ACKNOWLEDGEMENTS}

We thank Ms Anne Johnston and Ms Karen Jones from Sports Medicine Australia (WA
Branch) for coordination of the study. The Western Australian sports injury study was funded by the Western Australian Health Promotion Foundation (Healthway).

BrJ Sports Med 2003;37:380-381

\section{Authors' affiliations}

M Stevenson, Institute for International Health, University of Sydney, Sydney, Australia C Finch, NSW Injury Risk Management Research Centre, University of New South Wales

P Hamer, B Elliott, School of Human

Movement and Exercise Science, University of Western Australia

Correspondence to: Professor Stevenson, mstevenson@iih.usyd.edu.au

\section{REFERENCES}

1 Finch C, Owen N. Injury prevention and promoting physical activity: what is the nexus? J Sci Med Sport 2001;4:77-87.

2 Seward H, Orchard J, Hazard H, et al. Football injuries in Australia at the elite level. Med J Aust 1993;159:298-301.

3 Med J Aust 1993;159:298-301.

Participation in sport and physical activities, Australia. Canberra: ABS, 1998:Catalogue No 4177.0.

4 Stevenson M, Palamara P. Behavioural factors as predictors of motor vehicle crashes: differentials between young urban and rural difivers. Aust N Z J Public Health 2001;25:245-9

5 Van Mechelen W, Hlobil H, Kemper H. Incidence, severity, aetiology and prevention of sports injuries. Sports Med 1992;14:82-99.

6 Costa PT, Widger TA, eds. Personality disorders and the five-factor model of personality. Washington DC: American personality. Washington DC: Ame
Psychological Association, 1994.

7 Passer MW, Seese MD. Life stress and athletic injury: examination of positive versus negative events and three moderator variables. J Hum Stress 1983;4:11-16.

8 Sports Medicine Australia. Western Australian sports injury study. Perth, Western Australia: Sports Medicine Australia (WA Bunchlia: 2001

9 Stevenson $M$, Hamer $P$, Finch $C$, et al. Sport, age and sex specific incidence of sporting injuries in Western Australia. Br J Sports Med 2000;34: 188-94.

10 Finch C, Da Costa A, Stevenson M, et al. Sports injury experiences from the Western Australian sports injury cohort study. Aust N Z J Public Health 2002;26:462-7. 